ORIGINAL ARTICLE

\title{
The psychosocial work environment and alcohol dependence: a prospective study
}

\author{
J Head, S A Stansfeld, J Siegrist
}

Occup Environ Med 2004;61:219-224. doi: 10.1136/oem.2002.005256

See end of article for authors' affiliations

Correspondence to: Ms J Head, Department of Epidemiology and Public Health, University College London, London WCIE 6BT, UK; j.head@ucl.ac.uk

Accepted 5 June 2003

\begin{abstract}
Aims: To examine whether a stressful psychosocial work environment predicts alcohol dependence. Methods: Alcohol dependence of participants in the Whitehall II occupational cohort of London based civil servants (1985-88) was measured in 1991-93 using the CAGE questionnaire. The psychosocial work environment was measured by self report questions on the job demand-support-control model and on the model of effort-reward imbalance. Potential mediators including physical illness and poor mental health (GHQ) were measured at follow up in 1989.

Results: Effort-reward imbalance at work was associated with alcohol dependence in men after adjustment for employment grade and other baseline factors related to alcohol dependence. Although effort-reward imbalance predicted future longstanding illness, poor mental health and negative aspects of close relationships, the association between effort-reward imbalance and alcohol dependence in men was only partially mediated through these health and social support measures. In women, low decision latitude was related to alcohol dependence to some extent, but alcohol dependence among women was more prevalent in higher occupational grades. Men with high job demands or with low work social supports had a slightly reduced risk of alcohol dependence. No association was found between objectively assessed demands, job control, and alcohol dependence in either men or women.

Conclusion: A stressful psychosocial work environment in terms of effort-reward imbalance was found to be a risk factor for alcohol dependence in men. In view of the public health importance of alcohol dependence in working populations these findings call for more emphasis on psychosocial factors in occupational health research and prevention.
\end{abstract}

A lcohol dependence is a major public health issue in Western culture. It is associated with psychiatric complications, such as depression, as well as physical illness subsequent to high levels of alcohol intake. Alcohol dependence also has effects on social relationships and may influence performance at work and sickness absence from work.

The study of determinants of alcohol dependence in adults has largely focused on genetic and personality factors or on general socioeconomic conditions. There has been less research on the role of an adverse work environment. Several investigators following this line found some evidence of an association of shift work, ${ }^{1}$ low level of technical responsibility at work, ${ }^{2}$ and job insecurity ${ }^{3}$ with alcohol consumption. However, associations of an adverse work environment with alcohol use are often moderated by distinct coping characteristics of working people. ${ }^{3}$ Moreover, studies in this area are rarely based on an explicit stress-theoretical model that identifies "toxic" components of an adverse work environment, with special emphasis on its psychosocial dimensions.

The advent of two such models, the demand-supportcontrol model of job strain, ${ }^{5}$ and the effort-reward imbalance model, ${ }^{6}$ give new opportunities for testing an association of work conditions and alcohol dependence. In this paper, we examine associations between measures of these two models of a stressful psychosocial work environment and subsequent alcohol dependence in a prospectively designed investigation of a large cohort of civil servants.

\section{METHODS}

The Whitehall II study was set up to investigate the causes of the social gradient in morbidity and mortality. All nonindustrial civil servants aged 35-55 years working in the
London offices of 20 departments were invited to participate in the study. Between September 1985 and March 1988, participants attended a screening examination and completed a self report questionnaire (phase 1). The overall response rate was $73 \%$ ( $74 \%$ for men, $71 \%$ for women). The true response rates are likely to be higher, however, because around $4 \%$ of those on the list of employees had moved before the study and were not eligible for inclusion. Altogether 10308 civil servants were examined: 6895 men (67\%) and 3413 women (33\%). After initial participation at phase 1, a further postal questionnaire was carried out in 1989 (phase 2), and participants were approached again for a further screening examination in 1991-93 (phase 3). The participation rates at phase 2 and phase 3 were $79 \%$ and $83 \%$ respectively; 7372 subjects $(72 \%)$ were participants at all three of phases; 9302 participants (90\%) took part at either phase 2 or phase 3 . The length of follow up between phase 1 and phase 3 was 5.3 years, on average. Full details of the screening examinations have been reported elsewhere. ${ }^{7}$

Self report questionnaires have been administered at all phases, collecting information relating to personal characteristics, family, work environment, health behaviour, social support, and self reported health. Further details of non-work related risk factors for health are reported elsewhere. ${ }^{78}$

\section{Grade of employment}

Information on grade of employment was obtained by asking all participants to give their civil service grade title. The Civil Service identifies 12 non-industrial grade levels on the basis of salary. There was a steep increment in salaries from an annual salary in 1987 of between $£ 3061$ and $£ 5841$ in the clerical and office support grades to between $£ 18020$ and $£ 62100$ in the top unified grades $1-6$. Besides the steep increment in salaries there were also marked differences in 
Main messages

- Effort-reward imbalance at work was associated with alcohol dependence in men.

- In women, there was some association between decision latitude and alcohol dependence.

other socioeconomic indicators (education, housing tenure, car ownership, and fathers' occupation) by grade of employment. ${ }^{7}$ For analysis purposes, the 12 civil service grade levels were grouped into six categories.

\section{Work characteristics}

The job strain model developed by Karasek and Theorell ${ }^{5}$ posits that people working in jobs that are simultaneously characterised by high demands, low social support, and low control in terms of decision latitude are at risk of stress related ill health and disease. Decision latitude ( 15 items), psychological job demands (four items), and work social supports ( six items) were measured at all three phases using self completion questions derived from the Karasek and Theorell Job Content Instrument. ${ }^{5}$ Decision latitude can be subdivided into two components: decision authority, the amount of control over work; and skill discretion, a measure of job variety and opportunity for use of skills at work. Work social support has three components: support from colleagues; support from supervisors; and clarity and consistency of information from supervisors. Total scores for each of the three main constructs (decision latitude, job demands, work social supports) were computed; scores were then divided into tertiles labelled "low", "medium", or "high", so that, for example, those participants within the top third of all scores on the job demand scale were labelled as having "high job demands".

A different model of a stressful psychosocial work environment, the effort-reward imbalance model, conceptualises psychosocial stress at work in terms of an imbalance between high efforts spent and low rewards received. Lack of reciprocity between "costs" and "gains" in a core social role, the work role, is considered stressful, in particular if working people have no alternative choice. The full operationalisation of the model distinguishes two components of efforts: an extrinsic component reflecting job conditions such as demands and obligations and an intrinsic component reflecting personal coping style or overcommitment. ${ }^{6}$ The measure of effort-reward imbalance available for this analysis was derived using items included in the questionnaire at phase 1 where high efforts reflected the intrinsic rather than the extrinsic component of the model. ${ }^{10}$ High efforts were defined as high scores on one or more of the three subscales: competitiveness (three items), work related over-commitment (four items), or hostility (three items). Low rewards were defined by poor promotion prospects or a blocked career. Participants were classified into one of three groups: neither high efforts nor low rewards; one of high efforts or low rewards; both high effort and low rewards. This latter group was expected to exhibit highest levels of sustained stressful experience at work.

At phase 1, external assessments of job control and job demands were carried out by Civil Service personnel officers. ${ }^{11}$ In 18 of 20 departments in our study, 140 personnel managers assessed each job, independently of the holder of the post, for the level of control and work demands. In all, 5766 different jobs were rated covering 8838 participants.

\section{Policy implications}

- These findings call for more emphasis on psychosocial factors in occupational health.

- More research is needed on the link between psychosocial work characteristics and alcohol dependence.

\section{Alcohol dependence}

Alcohol dependence was measured by the CAGE questionnaire which was first included in the Whitehall II study at phase 3. This brief four item scale is a well used and validated screening instrument for alcohol dependence developed originally for general practice settings. The CAGE has been used in a variety of clinical settings ${ }^{12}$ and in population surveys. ${ }^{13-15}$ This short scale appears to correlate well with a clinical diagnosis of alcoholism ${ }^{16}$ and may even be a better predictor than biochemical indicators of alcohol dependence. ${ }^{17} \mathrm{~A}$ cut off of two or more positive responses to the CAGE questions has been found to identify problems with alcohol in a number of studies ${ }^{12}$ and has been used here.

\section{Statistical analysis}

Logistic regression was used to examine the association between phase 1 risk factors and alcohol dependence at phase 3. Results are presented as odds ratios with their $95 \%$ confidence intervals. First, the association between each of the work characteristics measures and alcohol dependence was analysed, adjusting for age and employment grade. Subsequent analyses adjusted for baseline health and nonwork predictors of alcohol dependence. As associations may be due to reporting bias, we also included a measure of negative affectivity. Potential non-work risk factors included health behaviours (smoking, alcohol consumption, physical activity), marital status, social supports outside work, material difficulties, and stressful life events. In this paper, we only present results for those non-work factors which were found to be associated with alcohol.

The original interpretation of the job strain model postulates that high job demands will have the greatest adverse effect on health in those with low decision latitude. In order to test this hypothesis, further analyses were run to test whether there were interactions between any of the work measures in their influence on health. Finally, analyses were repeated with interaction terms between employment grade and each of the work measures to test whether there is a differential effect of work related factors by socioeconomic status.

\section{RESULTS}

Table 1 shows the prevalence of alcohol dependence by employment grade for men and women. In women, there was a clear grade gradient with those in the highest two grades having the highest proportion of problem drinkers. This was not the case in men for whom there was little grade gradient.

Table 2 (model 1) shows the association between each of the work measures and alcohol dependence, after taking account of age and employment grade. In men, effort-reward imbalance was associated with alcohol dependence, with those classified as putting in high efforts but receiving low rewards having the highest risk of being alcohol dependent. This association was also seen for women, although was not as marked. In addition, low decision latitude in women was associated with increased risk of alcohol dependence.

Contrary to the original hypothesis neither high job demands nor low work support were associated with alcohol 
Table 1 Percentages of men and women classified as alcohol dependent at phase 3 by employment grade at phase 1 and age adjusted odds ratios $(95 \% \mathrm{Cl})$

\begin{tabular}{|c|c|c|c|c|}
\hline \multirow[b]{2}{*}{ Grade level $^{*}$} & \multicolumn{2}{|c|}{$\operatorname{Men}(n=5714)$} & \multicolumn{2}{|c|}{ Women $(n=2566)$} \\
\hline & $\%$ & Odds ratio $(95 \% \mathrm{Cl})$ & $\%$ & Odds ratio $(95 \% \mathrm{Cl})$ \\
\hline UG1-UG6 & 11 & 1 & 14 & 1 \\
\hline UG7 & 10 & $0.86(0.7$ to 1.1$)$ & 12 & 0.77 (0.4 to 1.5$)$ \\
\hline SEO & 12 & $1.02(0.8$ to 1.4$)$ & 10 & $0.63(0.3$ to 1.3$)$ \\
\hline HEO & 11 & 0.89 (0.7 to 1.2$)$ & 10 & $0.66(0.3$ to 1.3$)$ \\
\hline EO & 12 & 0.97 (0.7 to 1.3$)$ & 7 & $0.46(0.2$ to 0.9$)$ \\
\hline Clerical & 12 & 1.06 (0.7 to 1.5$)$ & 4 & $0.25(0.1$ to 0.5$)$ \\
\hline
\end{tabular}

${ }^{*}$ Civil Service unified grades $1-6$ and 7 consist of senior management and equivalent grades; SEO, HEO and EO consist of executive and equivalent professional grades; clerical represents the clerical and office support staff.

dependence. The interaction term of high demand and low control was not significantly related to alcohol dependence (results not shown).

As the CAGE questionnaire was not included at phase 1, we have taken units of alcohol consumption as an indicator of problem drinking at baseline. Other factors found to be associated with alcohol dependence were baseline health, smoking habits, physical exercise, height, negative aspects of close relationships, social networks, and negative affectivity (see table 3 ). Adjusting for these non-work factors accounted for some of the association between effort-reward imbalance and alcohol dependence (model 2, table 2), but for men there was still a significant association between effort-reward imbalance and alcohol dependence. The association between low decision latitude and alcohol dependence in women was hardly altered by adjustment for non-work factors, but was of borderline significance. Among men, an association between both job demands and work social supports emerged after adjustment for nonwork factors, but this was of borderline significance and not in the direction expected. Men with high job demands and low work social supports had a lower risk of alcohol dependence after adjustment for non-work factors. Some indicators of pattern of drinking (usual and maximum amount consumed on one occasion) were also included at phase 1. Additional adjustment for these measures did not alter the associations between work characteristics and alcohol dependence.

Following this, we additionally adjusted each work characteristic for the other work characteristics, testing whether the effects for individual work characteristics could be explained by the other work characteristics (model 3, table 2). As can be seen, an imbalance between high effort and low reward at work remains a significant predictor of alcohol dependence in men, but not in women. Low decision latitude remains associated with alcohol dependence in women to some extent, but not in men. Interestingly, high work demand and low work support, the other two components of the job strain model, show opposite effects on alcohol dependence among men (borderline significance).

There were no significant associations between externally assessed work characteristics and alcohol dependence (table 4). This holds true for job demands and for job control in both sexes. It is possible that this measure, although an external assessment of work, and thus potentially free from the bias inherently present in self report measures, did not capture those individual experiences of work which may be a risk factor for alcohol dependence.

The presence of baseline psychiatric disorder and baseline longstanding illness were both predictors of alcohol dependence at follow up after adjustment for age and employment grade (model 1, table 3 ). Similarly, both smoking and alcohol intake at baseline were powerful predictors of alcohol dependence at follow up, although lack of exercise was not a predictor. Negative aspects of close relationships and network size, after adjustment for smoking, were also predictive of alcohol dependence at follow up. After adjustment for work characteristics and other risk factors the baseline risk associated with psychiatric disorder was no longer significant, although was maintained for longstanding

Table 2 Odds ratios $(95 \% \mathrm{Cl})$ for alcohol dependence at phase 3 by work characteristics at phase 1, adjusted for age and employment grade (model 1), additionally adjusted for other predictors (model 2), and adjusted for other predictors and other self report work characteristics (model 3)

\begin{tabular}{|c|c|c|c|c|c|c|}
\hline & \multicolumn{3}{|l|}{ Men } & \multicolumn{3}{|l|}{ Women } \\
\hline & Model 1 & Model 2 & Model 3 & Model 1 & Model 2 & Model 3 \\
\hline Decision latitude & & $(n=4809)$ & & & $(n=1998)$ & \\
\hline High & 1 & 1 & 1 & 1 & 1 & 1 \\
\hline Medium & 1.19 (1.0 to 1.5$)$ & 1.12 (0.9 to 1.4$)$ & $1.13(0.9$ to 1.4$)$ & $1.14(0.7$ to 1.8$)$ & 1.13 (0.7 to 1.9$)$ & 1.14 (0.7 to 1.9$)$ \\
\hline Low & 1.20 (0.9 to 1.6$)$ & 1.03 (0.8 to 1.4$)$ & $1.02(0.8$ tol .4$)$ & 1.46 (0.9 to 2.4$)$ & 1.43 (0.8 to 2.5$)$ & $1.45(0.8$ to 2.6$)$ \\
\hline \multicolumn{7}{|l|}{ Job demands } \\
\hline Low & 1 & 1 & 1 & 1 & 1 & 1 \\
\hline Medium & 0.92 (0.7 tol.2) & 0.92 (0.7 tol.2) & 0.88 (0.7 to 1.1$)$ & 1.18 (0.8 to 1.8$)$ & $0.94(0.6$ to 1.5$)$ & 0.95 (0.6 to 1.5 ) \\
\hline High & $0.97(0.7$ to 1.3$)$ & $0.82(0.6$ to 1.1$)$ & $0.73(0.6$ to 1.0$)$ & $1.22(0.7$ to 2.0$)$ & $1.00(0.6$ to 1.7$)$ & 1.00 (0.6 to 1.8$)$ \\
\hline \multicolumn{7}{|l|}{ Work supports } \\
\hline High & 1 & 1 & 1 & 1 & 1 & 1 \\
\hline Medium & $0.90(0.7$ to 1.1$)$ & 0.83 (0.7 to 1.0$)$ & $0.80(0.6$ to 1.0$)$ & $1.09(0.7$ to 1.7$)$ & 1.01 (0.6 tol.6) & 0.99 (0.6 to 1.6$)$ \\
\hline Low & 1.03 (0.8 to 1.3 ) & 0.86 (0.7 to 1.1$)$ & 0.81 (0.6 tol.0) & $1.07(0.7$ to 1.6$)$ & 0.95 (0.6 tol. .5$)$ & $0.88(0.5$ to 1.4$)$ \\
\hline \multicolumn{7}{|c|}{ High effort, low reward } \\
\hline Neither & 1 & 1 & 1 & 1 & 1 & 1 \\
\hline Either & $1.69(1.2$ to 2.3$)$ & 1.44 (1.1 to 2.0$)$ & 1.56 (1.1 to 2.2$)$ & $1.15(0.6$ to 2.1$)$ & $1.11(0.6$ to 2.1$)$ & $1.11(0.6$ to 2.1$)$ \\
\hline Both & $2.26(1.6$ to 3.1$)$ & $1.69(1.2$ to 2.4$)$ & 1.93 (1.4 to 2.7$)$ & $1.63(0.9$ to 3.0$)$ & 1.25 (0.6 to 2.4$)$ & 1.25 (0.6 to 2.5$)$ \\
\hline
\end{tabular}

Model 2 includes adjustment for age, employment grade, GHQ, longstanding illness, smoking, and alcohol consumption (phase 1), exercise, height, negative affectivity, negative aspects of social supports, and network size. 
Table 3 Odds ratios $(95 \% \mathrm{Cl})$ for alcohol dependence at phase 3 by other risk factors at phase 1, adjusted for age and employment grade (model 1), and additionally adjusted for work characteristics and other risk factors (model 2)

\begin{tabular}{|c|c|c|c|c|}
\hline & \multicolumn{2}{|l|}{ Men $(n=4809)$} & \multicolumn{2}{|l|}{ Women $(n=1998)$} \\
\hline & Model 1 & Model 2 & Model 1 & Model 2 \\
\hline \multicolumn{5}{|l|}{ GHQ caseness } \\
\hline No & 1 & 1 & 1 & 1 \\
\hline Yes & $1.64(1.3$ to 2.0$)$ & 1.14 (0.9 to 1.4$)$ & 1.61 (1.1 to 2.3 ) & 1.17 (0.8 to 1.8$)$ \\
\hline \multicolumn{5}{|l|}{ Longstanding illness } \\
\hline No & 1 & 1 & 1 & 1 \\
\hline Yes & 1.84 (1.4 to 2.4$)$ & $1.43(1.1$ to 1.9$)$ & $1.51(0.9$ to 2.5$)$ & 1.23 (0.7 to 2.2$)$ \\
\hline Negative affectivity score & 1.18 (1.13 to 1.22$)$ & 1.11 (1.06 to 1.17 ) & $1.17(1.10$ to 1.24$)$ & 1.14 (1.05 to 1.23 ) \\
\hline \multicolumn{5}{|l|}{ Smoking } \\
\hline None & 1 & 1 & 1 & 1 \\
\hline Ex-smoker & 2.13 (1.7 to 2.6$)$ & $1.62(1.3$ to 2.0$)$ & 2.43 (1.6 to 3.6$)$ & 1.80 (1.2 to 2.8$)$ \\
\hline $1-10$ a day & 2.41 (1.6 to 3.6$)$ & $1.63(1.1$ to 2.5$)$ & $2.29(1.1$ to 4.6$)$ & $1.22(0.6$ to 2.6$)$ \\
\hline 11-20 a day & 1.65 (1.1 to 2.6$)$ & $0.97(0.6$ to 1.6$)$ & $2.19(1.2$ to 4.0$)$ & 1.69 (0.9 to 3.2$)$ \\
\hline $21+$ a day & 3.05 (2.0 to 4.6$)$ & 1.38 (0.9 to 2.2$)$ & $3.26(1.5$ to 7.3$)$ & 1.42 (0.6 to 3.5$)$ \\
\hline \multicolumn{5}{|l|}{ Units of alcohol M (W) } \\
\hline 0 & 0.88 (0.6 to 1.4$)$ & 0.84 (0.5 to 1.3$)$ & 0.67 (0.3 to 1.4$)$ & $0.67(0.3$ to 1.4$)$ \\
\hline $1-10(1-7)$ & & 1 & 1 & 1 \\
\hline $11-24(8-14)$ & $3.20(2.5$ to 4.2$)$ & $2.96(2.3$ to 3.9$)$ & 4.47 (2.7 to 7.3$)$ & $4.28(2.6$ to 7.1$)$ \\
\hline $25-35(15+)$ & 6.98 (5.3 to 9.2$)$ & $6.73(5.0$ to 9.0$)$ & 9.44 (5.7 to 15.7$)$ & $8.77(5.2$ to 14.9$)$ \\
\hline $36+$ & $10.72(8.0$ to 14.4$)$ & 9.47 (6.9 to 12.9$)$ & $14.47(5.0$ to 42.0$)$ & 11.65 (3.7 to 36.6$)$ \\
\hline \multicolumn{5}{|l|}{ Exercise } \\
\hline Vigorous & 1 & 1 & 1 & 1 \\
\hline Moderate & 0.92 (0.7 to 1.2$)$ & 0.93 (0.7 to 1.2 ) & 0.72 (0.4 to 1.2 ) & $0.72(0.4$ to 1.3$)$ \\
\hline Mild/none & 1.18 (0.9 to 1.5$)$ & 1.18 (0.9 to 1.5$)$ & 0.75 (0.4 to 1.3$)$ & $0.82(0.5$ to 1.4$)$ \\
\hline \multicolumn{5}{|c|}{ Negative aspects of close relationships } \\
\hline Low & 1 & 1 & 1 & 1 \\
\hline Medium & $1.30(1.0$ to 1.6$)$ & $1.17(0.9$ to 1.5$)$ & $1.02(0.7$ to 1.6$)$ & $0.86(0.5$ to 1.4$)$ \\
\hline High & 1.76 (1.4 to 2.2$)$ & $1.32(1.0$ to 1.7$)$ & $1.26(0.8$ to 1.9$)$ & $0.87(0.5$ to 1.4$)$ \\
\hline \multicolumn{5}{|l|}{ Network size } \\
\hline Low & 1 & 1 & 1 & 1 \\
\hline Medium & $0.77(0.6$ to 1.0$)$ & $0.71(0.6$ to 0.9$)$ & $0.80(0.5$ to 1.2$)$ & 0.69 (0.4 to 1.1$)$ \\
\hline High & 0.85 (0.7 to 1.1$)$ & 0.75 (0.6 to 1.0$)$ & $0.68(0.4$ to 1.1$)$ & $0.59(0.4$ to 0.9$)$ \\
\hline
\end{tabular}

illness in men but not in women (model 2, table 3 ). On the other hand, baseline alcohol intake remained a powerful predictor after adjustment for work characteristics and other risk factors. Smaller effects were also observed for negative affectivity and for smoking (borderline significance). Generally in men, but not in women, an effect of negative aspects of close relationships was still present for alcohol dependence at follow up after adjustment for work characteristics and other risk factors. A larger network size was found to be slightly protective of alcohol dependence in both sexes.

It may be that the observed associations between work characteristics and alcohol dependence are mediated through subsequent illness or change in social supports or social networks. We found that effort-reward imbalance and decision latitude predicted phase 2 measures of longstanding illness, mental health (GHQ) and negative aspects of close relationships, after adjustment for age, employment grade and baseline measures of GHQ, long standing illness, smoking, alcohol consumption, exercise, social supports, and network size (results not shown). However, effortreward imbalance and decision latitude were not related to subsequent social network size. In order to examine the possible mediating role of longstanding illness, mental health, and negative aspects of close relationships, further analyses of the associations between effort-reward imbalance, decision latitude, and alcohol dependence were carried out with and without adjustment for phase 2 measures of these potential mediators (table 5 ). The association between effort-reward imbalance and alcohol dependence in men was slightly reduced but remained significant after adjustment for the potential mediators. The association between decision latitude and alcohol dependence in women was hardly changed by adjustment for these potential mediators.

Table 4 Odds ratios $(95 \% \mathrm{Cl})$ for alcohol dependence at phase 3 by external work characteristics at phase 1, adjusted for age and employment grade (model 1), additionally adjusted for other predictors (model 2), and adjusted for other predictors and other externally assessed work characteristics (model 3)

\begin{tabular}{|c|c|c|c|c|c|c|}
\hline & \multicolumn{3}{|l|}{ Men } & \multicolumn{3}{|l|}{ Women } \\
\hline & Model 1 & Model 2 & Model 3 & Model 1 & Model 2 & Model 3 \\
\hline Externally assessed job control & & $(n=4261)$ & & & $(n=1808)$ & \\
\hline High & 1 & 1 & 1 & 1 & & 1 \\
\hline Medium & $0.83(0.7$ to 1.0$)$ & $0.89(0.7$ tol.1) & $0.88(0.7$ to 1.1$)$ & $1.28(0.8$ † 2.1$)$ & $1.26(0.8$ to 2.1$)$ & $1.24(0.7$ to 2.1$)$ \\
\hline Low & $0.86(0.6$ to 1.1$)$ & $0.91(0.7$ to 1.2$)$ & $0.88(0.6$ to 1.2$)$ & $0.87(0.5$ to 1.5$)$ & $1.07(0.6$ to 1.9$)$ & $1.00(0.5$ to 1.9$)$ \\
\hline \multicolumn{7}{|l|}{ Externally assessed job demands } \\
\hline Low & 1 & 1 & 1 & 1 & 1 & 1 \\
\hline Medium & $0.92(0.7$ to 1.2$)$ & $0.85(0.7$ tol. 1$)$ & $0.87(0.7$ to 1.1$)$ & $1.03(0.6$ to 1.7$)$ & $0.96(0.6$ to 1.6$)$ & $0.91(0.5$ to 1.5$)$ \\
\hline High & $0.98(0.8$ tol .2$)$ & 0.85 (0.7 tol.1) & 0.83 (0.6 tol.1) & 0.97 (0.6 tol .5$)$ & 0.84 (0.5 tol.4) & $0.83(0.5$ to 1.4$)$ \\
\hline
\end{tabular}

Model 2 includes adjustment for age, employment grade, GHQ, longstanding illness, smoking, and alcohol consumption (phase 1), exercise, height, negative affectivity, negative aspects of social supports, and network size. 
Table 5 Odds ratios $(95 \% \mathrm{Cl})$ for alcohol dependence at phase 3 by work characteristics at phase 1, before and after adjustment for potential mediators at phase 2

\begin{tabular}{|c|c|c|c|c|}
\hline & \multicolumn{2}{|l|}{$\operatorname{Men}(n=4259)$} & \multicolumn{2}{|l|}{ Women $(n=1729)$} \\
\hline & $\begin{array}{l}\text { Adjusted for age, employment } \\
\text { grade, and other phase } 1 \\
\text { predictors* }\end{array}$ & $\begin{array}{l}\text { Additionally adjusted } \\
\text { for potential mediators } \\
\text { at phase } 2 \dagger\end{array}$ & $\begin{array}{l}\text { Adjusted for age, } \\
\text { employment grade, } \\
\text { and other phase } 1 \\
\text { predictors* }\end{array}$ & $\begin{array}{l}\text { Additionally adjusted for } \\
\text { potential mediators at phase } 2 \dagger\end{array}$ \\
\hline \multicolumn{5}{|c|}{ Decision latitude } \\
\hline High & 1 & 1 & 1 & 1 \\
\hline Medium & $1.17(0.9$ to 1.9$)$ & 1.14 (0.9 to 1.5$)$ & $1.17(0.7$ to 2.0$)$ & $1.19(0.7$ to 2.0$)$ \\
\hline Low & $1.01(0.7$ to 1.4$)$ & 0.98 (0.7 to 1.4$)$ & $1.40(0.8$ to 2.5$)$ & $1.41(0.8$ to 2.6$)$ \\
\hline \multicolumn{5}{|c|}{ High effort, low reward } \\
\hline Neither & 1 & 1 & 1 & 1 \\
\hline Either & $1.49(1.1$ to 2.1$)$ & 1.38 (1.0 to 2.0$)$ & 1.17 (0.6 to 2.2 ) & $1.08(0.5$ to 2.1$)$ \\
\hline Both & $1.59(1.1$ to 2.3$)$ & $1.41(1.0$ to 2.0$)$ & 1.15 (0.6 to 2.3$)$ & $1.12(0.6$ to 2.3$)$ \\
\hline
\end{tabular}

*Other phase 1 predictors: GHQ, longstanding illness, smoking, alcohol consumption, exercise, height, negative affectivity, negative aspects of social supports, and network size.

†Phase 2 mediators: GHQ, longstanding illness, negative aspects of supports.

\section{DISCUSSION}

This contribution documents a striking association of adverse psychosocial work conditions in terms of effort-reward imbalance and alcohol dependence in men. This association remains significant after adjustment for relevant confounders, including concurrent psychosocial work characteristics. In addition, negative aspects of close relationships outside work and a small social network were also related to alcohol dependence, as was the experience of longstanding illness. Among women, low decision latitude at work to some extent was predictive of alcohol dependence. These associations between work characteristics and alcohol dependence did not appear to be mediated through physical illness, poor mental health, or adverse changes in social supports or network size. A social gradient of alcohol dependence was found among women, but not among men, with a higher prevalence of alcohol dependence among women in higher occupational grades. Neither objectively assessed psychosocial work conditions nor job strain, as originally hypothesised, were related to alcohol dependence. The fact that data are derived from a large prospective investigation and that a gender specific social gradient of alcohol consumption above recommended sensible levels is in line with results from contemporary national surveys, ${ }^{15} 18$ adds to the robustness of reported findings.

Most other studies of psychosocial work characteristics and alcohol have used measures of alcohol consumption rather than alcohol problems or alcohol dependence and most have found no or little association between work characteristics and amount consumed. ${ }^{19}$ A cross-sectional study of a French occupational cohort showed that, in men, low decision latitude was associated with alcohol consumption and, in women, low work social supports was associated with alcohol consumption. ${ }^{20}$

Effort-reward imbalance at work was found to be associated with a high level of alcohol consumption in a cross-sectional study. ${ }^{21}$ This observation further strengthens the validity of our results. Interestingly, experience of social reward deficiency has been hypothesised to act as one of several triggers activating the brain's dopaminergic reward system involved in addictive behaviour. ${ }^{22}$ Additional experience of an unfavourable relationship in private life, also related to alcohol dependence in our study, may reinforce a sense of social reward deficiency and related addictive behaviour. Perceived low control at work was related to alcohol dependence in women, but not in men. These findings call for additional investigations as low control at work is more prevalent in lower grades, whereas alcohol dependence was found to be more prevalent in higher grades in women.
Reported findings underline the usefulness of including self report measures of an adverse psychosocial work environment into research in occupational epidemiology, in addition to objectively assessed working conditions. Moreover, combining information from two main theoretical models of psychosocial stress at work, the job strain model and model of effort-reward imbalance, seems promising as this approach increases the explanatory power of work related predictors. In fact, several recent studies investigating the role of psychosocial work related stress with respect to different health outcomes have successfully applied this strategy. ${ }^{102324}$ However, several limitations of these reported results need to be taken into account. Firstly, it is not clear to what extent findings can be generalised beyond the current sample. The British Civil Service has traditionally been seen as a unique workforce with very high levels of job security and excellent working conditions. The degree to which findings from this workforce can be generalised to other occupational groups has been questioned. In fact, the Civil Service is not very different from other large white collar workforces, and the changes in the Civil Service in the past 10 years have made it more similar to other white collar workforces in both the public and private sectors. Clearly, blue collar and low status occupational conditions are underrepresented in the investigated population. As alcohol consumption is strongly patterned by sociocultural norms and values, its prevalence and its potential role in work related health may vary across societies. The prevalence of alcohol dependence among men was similar to the reported prevalence of $8-10 \%$ from national surveys of the British general population, but we found a higher overall prevalence of alcohol dependence among women than seen in national surveys where the estimated prevalence was around $2-4 \% .^{15} 25$

A second limitation concerns the assessment of alcohol dependence, using the CAGE questionnaire. The validity of self report measures of alcohol consumption has been questioned, referring to the social desirability effects of underreporting and more recently the tendency to over report on the CAGE in relation to increasing social intolerance of heavy drinking in Canada. ${ }^{26}$ It is not clear whether these findings are generalisable to the United Kingdom, and several investigations have found a high validity of CAGE questionnaire items ${ }^{16}{ }^{17}$ in a variety of cultural groups. ${ }^{13} 27$

Thirdly, our measure of effort-reward imbalance at work was not fully identical with the original measurement. However, core notions of the model are reflected in our operationalisation. Thus, rather than overestimating effects it 
is reasonable to assume that our restricted measure may underestimate an association of effort-reward imbalance at work with alcohol dependence.

A further limitation of our findings relates to the exclusive study of alcohol dependence. We did not explore additional forms of addictive behaviour, and thus were not able to study clusters of addictive behaviour in different groups of civil servants.

Despite these limitations our results underline the importance of adverse psychosocial work conditions, as identified by theoretical models, in predicting alcohol dependence. To our knowledge, this is the first prospectively documented evidence of effort-reward imbalance at work as a risk factor for alcohol dependence in men. This combination of putting in a lot of effort at work but receiving low rewards in terms of being valued, income, and promotion seems to be a potent risk factor for other health outcomes in the Whitehall II study as well, including coronary heart disease, ${ }^{10}$ psychiatric disorders, ${ }^{24}$ and health functioning, ${ }^{28}$ and in other investigations..$^{29} 30$

In conclusion, the association between work characteristics and alcohol dependence is a much under researched area and deserves further attention, not least because the work environment is a practical setting for interventions to prevent ill health.

\section{ACKNOWLEDGEMENTS}

We thank all participating civil service departments and their welfare, personnel, and establishment officers; the Council of Civil Service Unions; all participating civil servants in the Whitehall II study; Professor Sir Michael Marmot, Director of the Whitehall II study, and all members of the Whitehall II study team. We also thank two reviewers for their helpful comments on an earlier version of this paper.

The work presented in this paper was supported by a grant from the Health and Safety Executive. The Whitehall II study has been supported by grants from the Medical Research Council, the Economic and Social Research Council, Health and Safety Executive, Department of Health, British Heart Foundation, National Heart Lung and Blood Institute (HL36310), National Institute on Aging (AG13196), Agency for Health Care Policy Research (HS06516), and the John D and Catherine T MacArthur Foundation Research Network on Socioeconomic Status and Health.

\section{Authors' affiliations}

J Head, Department of Epidemiology and Public Health, University College London, UK

S A Stansfeld, Department of Psychiatry, Institute of Community Health Sciences, Barts and the London, Queen Mary's School of Medicine and Dentistry, Queen Mary, London, UK

J Siegrist, Department of Medical Sociology, University of Duesseldorf P.B. 101007 40001, Dusseldorf, Germany

\section{REFERENCES}

1 Tasto D, Collegan MJ, Skjei EW, et al. Health consequences of shift work. Manlow Park: National Institute of Occupational Health and Safety, 1978.

2 Plant MA. Drinking careers: occupations, drinking habits and drinking problems. London: Tavistock, 1979.
3 Cooper ML, Russell M, Frone MR. Work stress and alcohol effects: a test of stress-induced drinking. J Health Soc Behav 1990;31:260-76.

4 Mensch BS, Kandel DB. Do job conditions influence the use of drugs? J Health Soc Behav 1988;29:169-84.

5 Karasek R, Theorell T. Healthy work: stress, productivity, and the reconstruction of working life. New York: Basic Books, 1990.

6 Siegrist J. Adverse health effects of high-effort/low-reward conditions. J Occup Health Psychol 1996;1:27-41.

7 Marmot MG, Davey Smith G, Stansfeld SA, et al. Health inequalities among British Civil Servants: the Whitehall II study. Lancet 1991;337:1387-93.

8 Stansfeld SA, Fuhrer R, Shipley M. Types of social support as predictors of psychiatric morbidity in a cohort of British Civil Servants (Whitehall II Study). Psychol Med 1998:28:881-92.

9 Bosma $\mathrm{H}$, Marmot MG, Hemingway $\mathrm{H}$, et al. Low job control and risk of coronary heart disease in the Whitehall II (prospective cohort) study. BMJ 1997; 314:558-65.

10 Bosma H, Peter R, Siegrist J, et al. Two alternative job stress models and the risk of coronary heart disease. Am J Public Health 1998;88:68-74.

11 North FM, Syme SL, Feeney A, et al. Psychosocial work environment and sickness absence among British civil servants: the Whitehall II Study. Am J Public Health 1996;86:332-40.

12 Ewing JA. Detecting alcoholism, the CAGE questionnaire. JAMA 1984;252:1905-7.

13 Smart RG, Adlaf EM, Knoke D. Use of the CAGE scale in a population survey of drinking. J Stud Alcohol 1991;52:593-6.

14 Whichelow MJ. Trends in alcohol consumption. In: Cox BD, Huppert FA, Whichelow MJ, eds. Health and lifestyle survey: seven years on. Aldershot: Dartmouth, 1993.

15 Hedges B. Alcohol consumption. In: Colhoun $\mathrm{H}$, Prescott-Clarke P, eds. Health survey for England 1994. London: HMSO, 1996.

16 Mayfield D, McLeod G, Hall P. The CAGE questionnaire: validation of a new alcoholism screening instrument. Am J Psychiatry 1974;131:1121-3.

17 Bernadt MW, Mumford J, Taylor C, et al. Comparison of questionnaire and laboratory tests in the detection of excessive drinking and alcoholism. Lancet 1982;1:325-8.

18 Drever F, Whitehead M. Health inequalities, Decennial Supplement. Series DS No. 15, 1-257. London: The Stationery Office, Office for National Statistics, 1997.

19 Van Loon AJ, Tiihuis M, Surtees PG, et al. Lifestyle risk factors for cancer: the relationship with psychosocial work environment. Int I Epidemiol 2000;29:785-92

20 Niedhammer I, Goldberg M, Leclerc A, et al. Psychosocial work environment and cardiovascular risk factors in an occupational cohort in France. $J$ Epidemiol Community Health 1998;52:93-100.

21 Puls W, Winold $\mathrm{H}$, Blank T. The influence of effort-reward imbalance in the workplace on the consumption of alcohol: a written survey carried out in metal-working companies. Sucht 1998;44:183-99.

22 Siegrist J. Place, social exchange and health: proposed sociological framework. Soc Sci Med 2000;51:1283-93.

23 Peter R, Siegrist J, Hallqvist J, et al. Psychosocial work environment and myocardial infarction: improving risk estimation by combining two complementary job stress models in the SHEEP Study. J Epidemiol Community Health 2002;56:294-300.

24 Stansfeld SA, Fuhrer R, Shipley MJ, et al. Work characteristics predict psychiatric disorder: prospective results from the Whitehall II study. Occup Environ Med 1999; 15:302-7.

25 Meltzer H, Gill B, Petticrew M, et al. OPCS surveys of psychiatric morbidity in Great Britain. The prevalence of psychiatric morbidity among adults living in private households. Report 1. London: HMSO, 1995:1-174.

26 Bisson J, Nadeau L, Demers A. The validity of the CAGE scale to screen for heavy drinking and drinking problems in a general population survey. Addiction 1999:94:715-22

27 Pileire B, Bredent-Bangou J, Valentino M. Comparison of questionnaire and biochemical markers to detect alcohol abuse in a West Indian population. Alcohol Alcohol 1991;26:353-9.

28 Stansfeld S, Bosma H, Hemingway $\mathrm{H}$, et al. Psychosocial work characteristics and social support as predictors of SF-36 functioning: the Whitehall II study. Psychosom Med 1998:60:247-55.

29 Marmot M, Siegrist J, Theorell T, et al. Health and the psychosocial environment at work. In: Marmot M, Wilkinson RG, eds. Social Determinants of Health. New York: Oxford University Press, 1999:105-31.

30 Schnall P, Belkic K, Landsbergis $\mathrm{P}$, et al. The workplace and cardiovascular disease. Occupational Medicine State of the Art Reviews 2000;15:1-334. 